\title{
SUPPLEMENTAL INFORMATION FOR WATER USE AND MANAGEMENT IN THE BAKKEN SHALE OIL PLAY IN NORTH DAKOTA
}

R.M. Horner, ${ }^{a, *}$ C.B. Harto, ${ }^{b}$ R.B. Jackson, ${ }^{c}$ E.R. Lowry, ${ }^{d}$ A.R. Brandt, ${ }^{\text {e }}$ T.W. Yeskoo, ${ }^{d}$ D.J. Murphy, ${ }^{f}$ and C.E. Clark ${ }^{\mathrm{b}}$

a U.S. Department of Energy, 1000 Independence Ave SW, Washington, DC 20585; 202-5868447; robert.horner@hq.doe.gov

b Environmental Science Division, Argonne National Laboratory, 955 L'Enfant Plaza SW, Suite 6000, Washington, DC 20024

c School of Earth, Energy, and Environmental Sciences, Woods Institute for the Environment, and Precourt Institute for Energy, Stanford University, Stanford, CA 94305

d School of Earth, Energy, and Environmental Sciences, Stanford University, Stanford, CA 94305

e School of Earth, Energy, and Environmental Sciences and Precourt Institute for Energy, Stanford University, Stanford, CA 94305

f Saint Lawrence University, 23 Romoda Drive, Canton, NY 13617 


\section{TABLE OF CONTENTS}

FIGURE S1 2008 Hydraulic Fracturing Locations and Water Use by HUC-8 Subbasin in the North Dakota Bakken Shale Play

FIGURE S2 2009 Hydraulic Fracturing Locations and Water Use by HUC-8 Subbasin in the North Dakota Bakken Shale Play

FIGURE S3 2010 Hydraulic Fracturing Locations and Water Use by HUC-8 Subbasin in the North Dakota Bakken Shale Play

FIGURE S4 2011 Hydraulic Fracturing Locations and Water Use by HUC-8 Subbasin in the North Dakota Bakken Shale Play

FIGURE S5 2012 Hydraulic Fracturing Locations and Water Use by HUC-8 Subbasin in the North Dakota Bakken Shale Play

TABLE S1 Estimated EUR for Bakken Shale Wells (bbl) ............................................... S-8

TABLE S2 2012 USGS Stream Flow by HUC-8 Subbasin Compared to Water Demand for Hydraulic Fracturing

TABLE S3 Produced Water TDS Levels from Major U.S. Shale Plays

REFERENCES. 


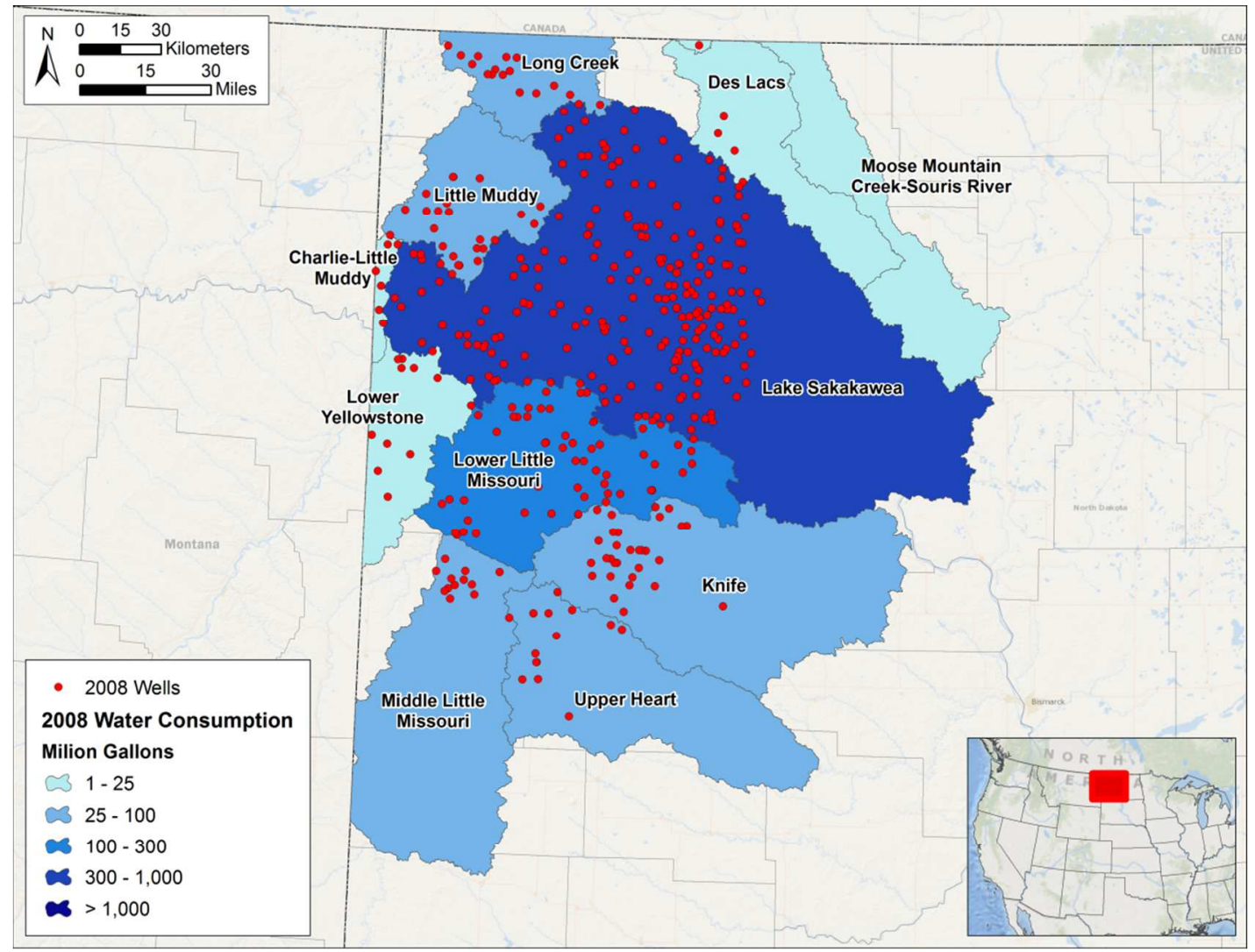

FIGURE S1 2008 Hydraulic Fracturing Locations and Water Use by HUC-8 Subbasin in the North Dakota Bakken Shale Play 


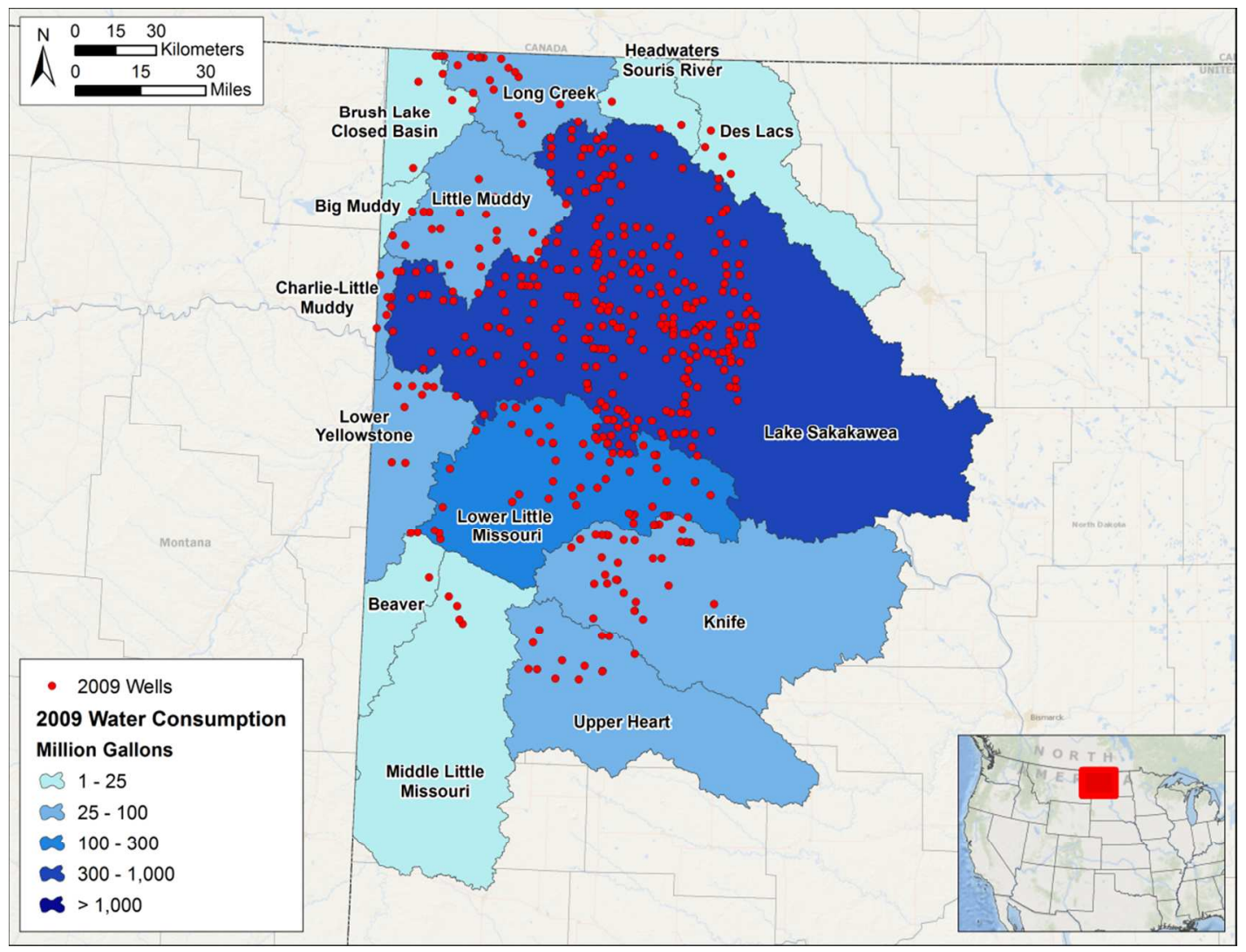

FIGURE S2 2009 Hydraulic Fracturing Locations and Water Use by HUC-8 Subbasin in the North Dakota Bakken Shale Play 


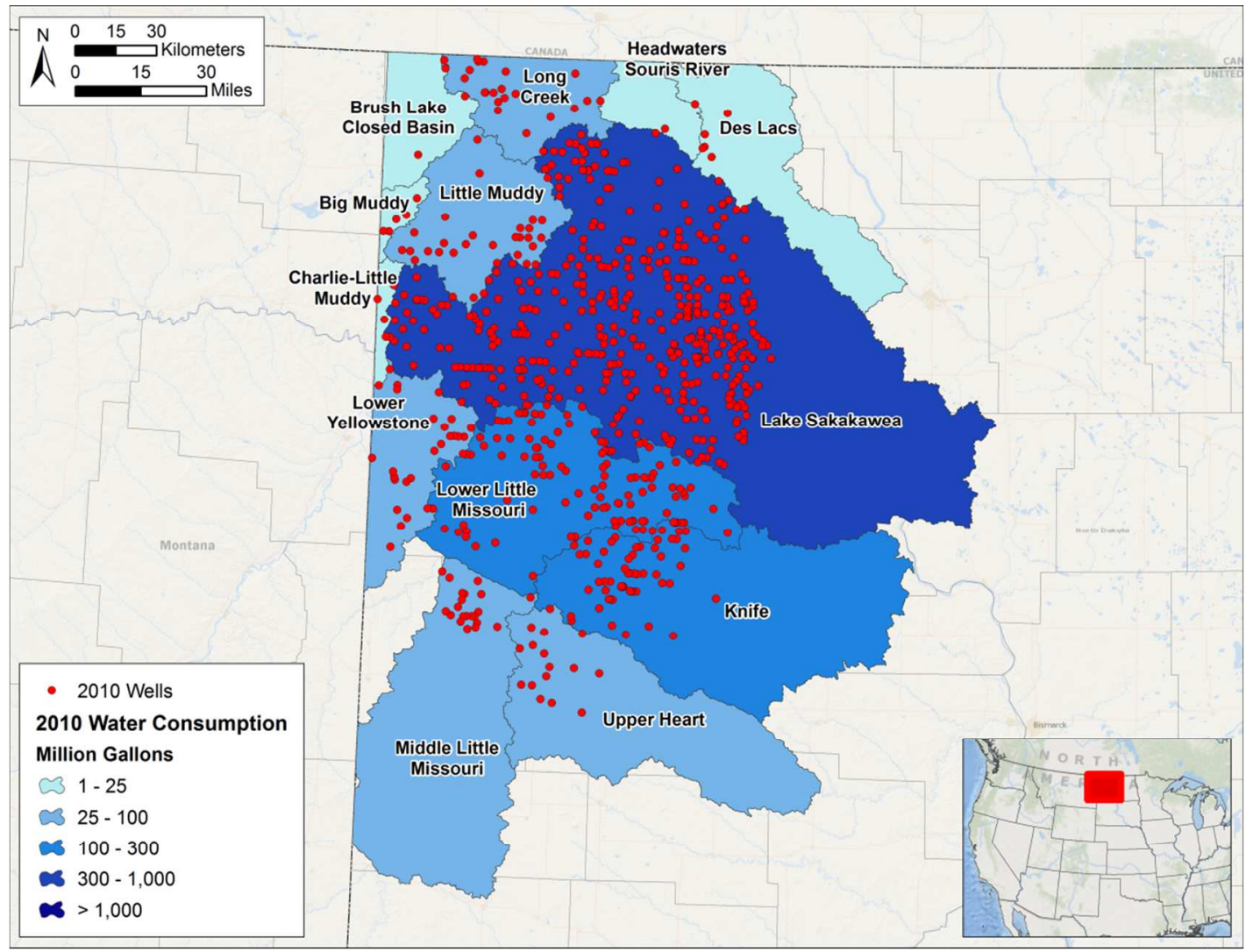

FIGURE S3 2010 Hydraulic Fracturing Locations and Water Use by HUC-8 Subbasin in the North Dakota Bakken Shale Play 


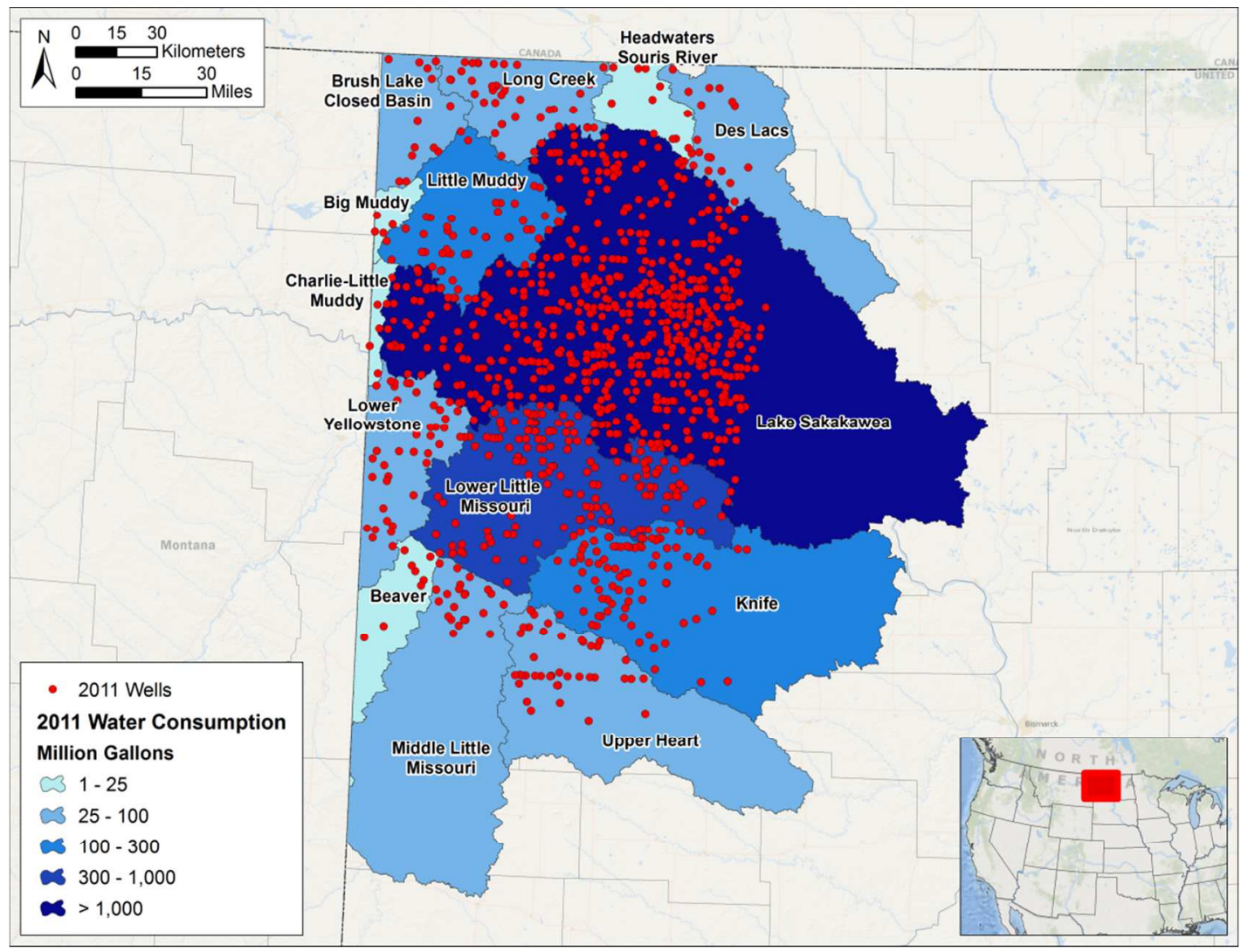

FIGURE S4 2011 Hydraulic Fracturing Locations and Water Use by HUC-8 Subbasin in the North Dakota Bakken Shale Play 


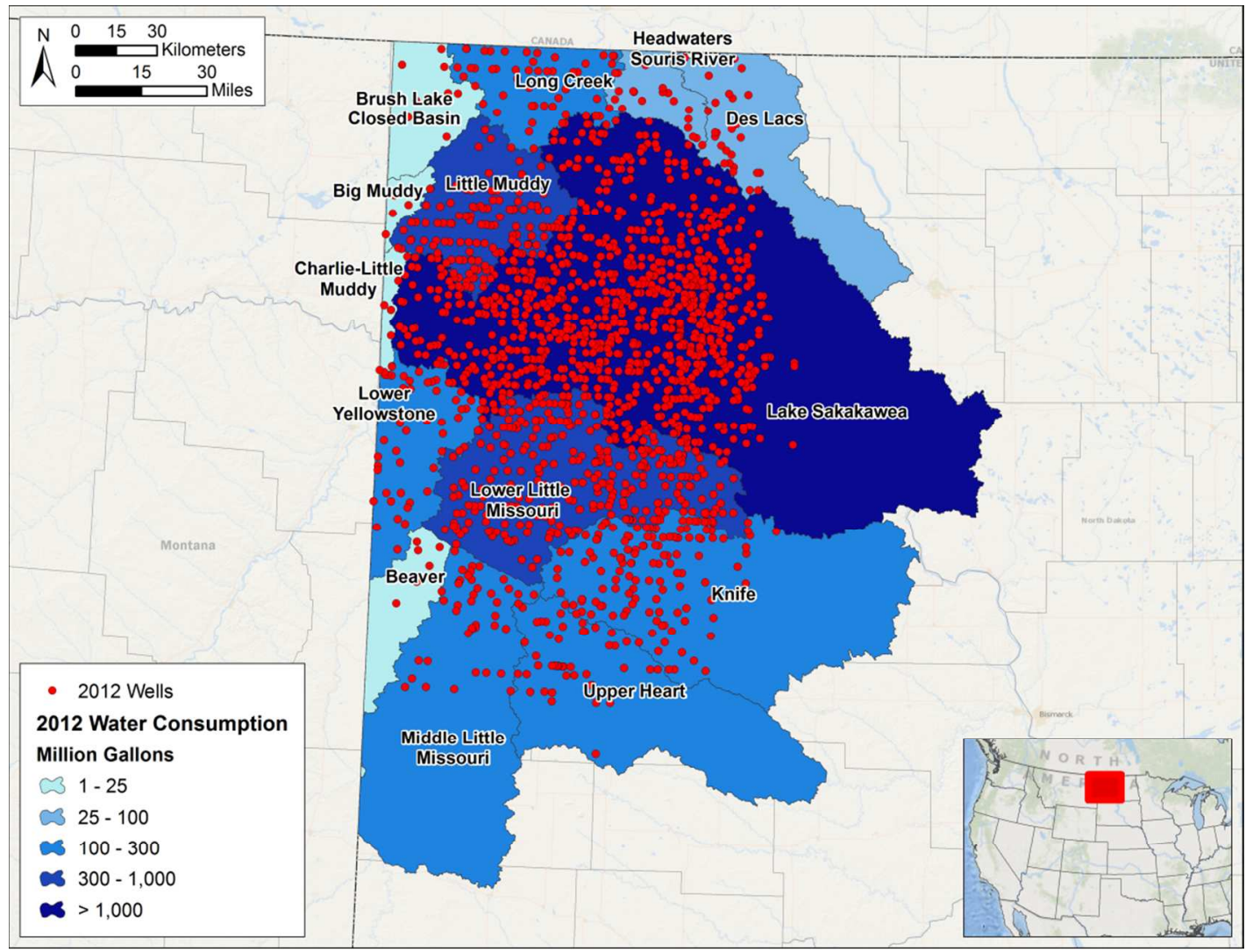

FIGURE S5 2012 Hydraulic Fracturing Locations and Water Use by HUC-8 Subbasin in the North Dakota Bakken Shale Play 
TABLE S1 Estimated EUR for Bakken Shale Wells (bbl)

\begin{tabular}{|c|c|c|c|c|c|c|}
\hline $\begin{array}{l}\text { Decline } \\
\text { Method }\end{array}$ & $\begin{array}{c}\text { 15-Year } \\
\text { Mean }\end{array}$ & $\begin{array}{l}\text { 15-Year } \\
\text { Median }\end{array}$ & $\begin{array}{c}15-\text { Year } \\
\text { Standard } \\
\text { Deviation }\end{array}$ & $\begin{array}{c}\text { 30-Year } \\
\text { Mean }\end{array}$ & $\begin{array}{l}\text { 30-Year } \\
\text { Median }\end{array}$ & $\begin{array}{c}\text { 30-Year } \\
\text { Standard } \\
\text { Deviation }\end{array}$ \\
\hline Hyperbolic & 270,000 & 221,000 & 198,000 & 334,000 & 252,000 & 280,000 \\
\hline $\begin{array}{l}\text { Stretched } \\
\text { Exponential }\end{array}$ & 231,000 & 181,000 & 194,000 & 270,000 & 190,000 & 295,000 \\
\hline
\end{tabular}

${ }^{a}$ Data sourced from McNally and Brandt (2015). ${ }^{1}$ 
TABLE S2 2012 USGS Stream Flow by HUC-8 Subbasin Compared to Water Demand for Hydraulic Fracturing

\begin{tabular}{lccc}
\hline \multicolumn{1}{c}{ HUC-8 Subbasin } & $\begin{array}{c}\text { Demand } \\
\text { (gal) }\end{array}$ & $\begin{array}{c}\text { Stream } \\
\text { Flow (gal) }\end{array}$ & $\begin{array}{c}\text { Demand as a } \\
\text { Percentage of } \\
\text { Surface Flow }\end{array}$ \\
\hline Beaver & & & \\
Big Muddy & $1.49 \mathrm{E}+07$ & $3.16 \mathrm{E}+09$ & $0.47 \%$ \\
Brush Lake Closed Basin & $2.03 \mathrm{E}+07$ & $3.22 \mathrm{E}+10$ & $0.06 \%$ \\
Charlie-Little Muddy & $1.99 \mathrm{E}+07$ & $1.96 \mathrm{E}+10$ & $0.10 \%$ \\
Des Lacs & $2.26 \mathrm{E}+07$ & $3.75 \mathrm{E}+10$ & $0.06 \%$ \\
Headwaters Souris River & $5.98 \mathrm{E}+07$ & $6.09 \mathrm{E}+09$ & $0.98 \%$ \\
Knife & $4.33 \mathrm{E}+07$ & No Data & - \\
Lake Sakakawea & $2.66 \mathrm{E}+08$ & $1.90 \mathrm{E}+10$ & $1.40 \%$ \\
Little Muddy & $2.24 \mathrm{E}+09$ & $8.31 \mathrm{E}+10$ & $2.70 \%$ \\
Long Creek & $3.02 \mathrm{E}+08$ & $4.84 \mathrm{E}+09$ & $6.25 \%$ \\
Lower Little Missouri & $1.16 \mathrm{E}+08$ & No Data & - \\
Lower Yellowstone & $6.99 \mathrm{E}+08$ & $2.07 \mathrm{E}+10$ & $3.37 \%$ \\
Middle Little Missouri & $1.57 \mathrm{E}+08$ & $1.78 \mathrm{E}+11$ & $0.09 \%$ \\
Upper Heart & $1.14 \mathrm{E}+08$ & $7.58 \mathrm{E}+09$ & $1.51 \%$ \\
\hline a Source: USGS (2013). ${ }^{2}$ & $1.08 \mathrm{E}+08$ & $9.97 \mathrm{E}+09$ & $1.08 \%$ \\
\hline & & &
\end{tabular}


TABLE S3 Produced Water TDS Levels from Major U.S. Shale Plays

\begin{tabular}{|c|c|}
\hline Shale Play & TDS $(\mathrm{mg} / \mathrm{L})$ \\
\hline \multirow[t]{3}{*}{ Barnett } & $60,000^{\mathrm{a}}$ \\
\hline & $500-200,000^{b}$ \\
\hline & $50,000-140,000^{c}$ \\
\hline \multirow[t]{2}{*}{ Bakken } & $35,000-400,000^{\mathrm{d}}$ \\
\hline & $150,000-219,000^{\mathrm{e}}$ \\
\hline \multirow[t]{3}{*}{ Fayetteville } & $25,000^{\mathrm{a}}$ \\
\hline & $3,000-80,000^{\mathrm{b}}$ \\
\hline & $15,000^{\mathrm{c}}$ \\
\hline \multirow[t]{2}{*}{ Haynesville } & $120,000^{\mathrm{a}}$ \\
\hline & $500-250,000^{b}$ \\
\hline \multirow[t]{3}{*}{ Marcellus } & $180,000^{\mathrm{a}}$ \\
\hline & $10,000-300,000^{b}$ \\
\hline & $40,000->120,000^{c}$ \\
\hline Woodford & $110,000^{\mathrm{a}}$ \\
\hline \multicolumn{2}{|c|}{ a Nolen $(2011) .^{3}$} \\
\hline \multicolumn{2}{|c|}{ b Alleman (2011). ${ }^{4}$} \\
\hline \multicolumn{2}{|c|}{${ }^{c}$ Mantell (2011). ${ }^{5}$} \\
\hline \multicolumn{2}{|c|}{${ }^{d}$ Shaffer et al. (2013). ${ }^{6}$} \\
\hline \multicolumn{2}{|c|}{${ }^{\mathrm{e}}$ Stepan et al. (2010). ${ }^{7}$} \\
\hline
\end{tabular}




\section{REFERENCES}

(1) McNally, M. S.; Brandt, A. R. The productivity and potential future recovery of the Bakken formation of North Dakota. Journal of Unconventional Oil and Gas Resources, 2015, 11, 11-18.

(2) Water Watch, U.S. Geological Survey, 2013; http://waterwatch.usgs.gov.

(3) Nolen, C. H. Water Management in Unconventional Natural Gas Exploration and Production: An Important and Long-Term Challenge, NJWEA Annual Conference, May 2011; http://www.aaees.org/downloadcenter/Presentation-NJWEA052011-CHunterNolen.pdf.

(4) Alleman, D. Treatment of Shale Gas Produced Water for Discharge. Water Resources Management. 2011, March 29-30;

http://www2.epa.gov/sites/production/files/documents/17_Alleman_-_Produced_Water_508.pdf.

(5) Mantell, M.E., 2011, Produced Water Reuse and Recycling Challenges and Opportunities Across Major Shale Plays, EPA Hydraulic Fracturing Study Technical Workshop \#4: Water Resources Management, March 29-30, available at: http://www2.epa.gov/sites/production/files/documents/09_Mantell_-_Reuse_508.pdf.

(6) Shaffer, D. L.; Chavez, L. H. A.; Ben-Sasson, M.; Castrillon, S. R. V.; Yip, N. Y.; Elimelech, M. Desalinization and Reuse of High-Salinity Shale Gas Produced Water: Drivers, Technologies, and Future Directions. Environmental Science and Technology, 2013, 47, 95699583.

(7) Stepan, D. J.; Shockey, R. E.; Kurz, B. A.; Kalenze, N. S.; Cowan, R. M.; Ziman, J. J.; Harju, J.A. Bakken Water Opportunities Assessment-Phase 1, prepared for North Dakota Industrial Commission by Energy and Environmental Research Center, University of North Dakota, 2010; http://www.undeerc.org/Water/pdf/FracWaterPhaseIreport.pdf. 